\title{
PROSTATITIS CRÓNICA: UNA REVISIÓN CRÍTICA DE SU ACTUAL DEFINICIÓN NOSOLÓGICA, CLASIFICACIÓN Y POTENCIAL CARCINOGÉNESIS.
}

\author{
Remigio Vela Navarrete, Carmen González Enguita, Juan Vicente García Cardoso, \\ G. Manzarbeitia y F. Soriano García.
}

Cátedra de Urología de la Universidad Autónoma de Madrid. Servicio de Urología de la Fundación Jiménez Díaz. Departamentos de Anatomía Patológica y Microbiología de la Fundación Jiménez Díaz. Madrid. España.

\begin{abstract}
Resumen.- Revisión crítica actualizada de la prostatitis crónica, como entidad nosológica, anatomoclínica, supuestamente de origen microbiológico o inflamatorio. Argumentación científica, a la luz de los nuevos progresos, sobre el papel de la inflamación amicrobiana, tanto a nivel de la próstata craneal como de la caudal, para reconsiderar la conveniencia de mantener la clasificación actual de las prostatitis crónicas, y especialmente el apartado referido a la "prostatitis histológica". Análisis de evidencias relacionando la prostatitis "con el dolor pélvico", síndrome dominante en muchos pacientes y fundamento de la actual propuesta terminológica; prostatitis-dolor pélvico. Papel de la inflamación en la génesis de la HBP y cáncer de próstata. Justificación y conveniencia de un nuevo consenso terminológico sobre las prostatitis, en general.
\end{abstract}

Palabras clave: Prostatitis crónica. Inflamación prostática. Dolor pélvico.

Summary.- Updated critical review of chronic prostatitis as a nosologic, anatomic-clinical entity of supposed microbiological or inflammatory origin. Scientific reasoning about the role of amicrobial inflammation in both caudal and cranial prostate, after new progresses, to reconsider the convenience of maintaining the current classification of chronic prostatitis, mainly in the section referred to "histological prostatitis". Analysis of scientific evidences relating prostatitis and "pelvic pain", the dominant syndrome in many patients and basement of the current terminological proposal: prostatitis-pelvic pain. The role of inflammation in the genesis of $\mathrm{BPH}$ and prostate cancer. Justification and convenience of a new term in logic consensus on prostatitis.

Keywords: Chronic prostatitis. Prostatic inflammation. Pelvic pain.

\section{INTRODUCCIÓN}

Cuando nos enfrentamos al inconfundible cuadro clínico de la prostatitis aguda, con el riguroso cumplimiento de los postulados exigidos por la medicina anatomoclínica en los procesos de etiología bacteriana, con evidentes signos y síntomas de focalidad, junto a síndrome febril llamativo, evidencias analíticas y microbiológicas de infección y respuesta satisfactoria al tratamiento con antimicrobianos, la prostatitis crónica, por el contrario, se muestra, desde que este término fue introducido en clínica, como un proceso de límites nosológicos confusos, de etio- 
logía indeterminada, englobando bajo su definición enfermos con un perfil clínico muy variado en los que suele dominar el carácter incómodo y doloroso del proceso, fundamentalmente referido al área perineal, sobre signos y síntomas genuinamente infectivos. Y sucede todo ello a pesar de los enormes esfuerzos realizados en los últimos años para desentrañar los misterios microbiológicos y patrones anatomoclínicos ocultos detrás de un término de tan extraordinaria ambigüedad.

En los comentarios que siguen pretendemos hacer una valoración crítica de los últimos progresos conceptuales sobre éste padecimiento, que han motivado una nueva clasificación de las prostatitis, con innovaciones terapéuticas aún de dudosa eficacia, incluyendo un análisis de sospechas y evidencias relacionadas con el potencial efecto carcinogénico de la inflamación prostática o su relación con la hiperplasia prostática. Quizás deba presidir esta revisión una observación clínica incontestable; rara vez los pacientes diagnosticados de prostatitis crónica, no bacteriana, tienen en su historia clínica un episodio previo, rotundo, de prostatitis aguda.

\section{Perfil clínico y clasificaciones de las prostá- titis crónicas}

Constituye una referencia obligada el trabajo de Stamey de finales de los 60 , publicado en 1972 (1) para aclarar los motivos que han guiado a los urólogos para determinar la patogenia de la prostatitis crónica, desde la más que razonable sospecha de que no todas tenían un fundamento etiológico bacteriano. De este modo, el objetivo inicial fue comprobar en cuantos pacientes existían argumentos y evidencias de un proceso infectivo y en cuántos de ellos, en ausencia de infección demostrable, existía un proceso inflamatorio, revelado por alteraciones reconocibles en la secreción prostática, tras un masaje intenso, que permitía la identificación, al microscopio, especialmente el microscopio de contraste de fases, de un mayor número de leucocitos que en individuos normales. Las cuestiones que se debatían pueden resumirse en estas dos preguntas: ¿̇son todas las prostatitis crónicas infecciosas o motivadas por un proceso inflamatorio de otra naturaleza?; jes el dolor pélvico acompañante, y a veces el más relevante síntoma de estos procesos, de origen prostático?

A la primera pregunta intentaron contestar Stamey y Meares (2) mediante el contaje diferenciado de bacterias y leucocitos en muestras secuenciales, cada una de ellas representativa de una zona de la vía urinaria inferior, del siguiente modo: las muestras iniciales (M1 y $M 2$ ) corresponden a unos centímetros cúbicos de orina obtenidos en la fase inicial de la micción; la muestra siguiente (M3) representa la secreción obtenida después de un intenso masaje prostático; la siguiente muestra (M4) se obtiene de la micción que se realiza después del masaje. El resultado de estos estudios diferenciados de muestras secuenciales, se basaba, más que en valores absolutos de recuentos bacterianos o de leucocitos, en las diferencias cuantitativas observadas en las diferentes

TABLA I. CLASIFICACION DE LAS PROSTATITIS.

\begin{tabular}{|c|c|c|c|}
\hline DRACH 1978 & & & NIH 1995 \\
\hline TERMINOLOGÍA & & & NUEVA TERMINOLOGÍA \\
\hline Prostatitis bacteriana aguda & I & I & Prostatitis bacteriana aguda \\
\hline Prostatitis bacteriana crónica & II & II & Prostatitis bacteriana crónica \\
\hline Prostatitis crónica abacteriana & III & $\begin{array}{l}\text { III } \\
\text { IIla } \\
\text { IIlb }\end{array}$ & $\begin{array}{l}\text { Síndrome P. crónica/Dolor pélvico crónico } \\
\text { con inflamación } \\
\text { sin inflamación }\end{array}$ \\
\hline Prostatodinia & IV & IV & $\begin{array}{l}\text { Prostatitis inflamatoria asintomática } \\
\text { (prostatitis histológica }\end{array}$ \\
\hline
\end{tabular}


muestras, asumiéndose que un número mayor de bacterias o leucocitos en las últimas nuestras orientaban hacia un proceso infeccioso, simplemente inflamatorio, o ausencia de cualquier inflamación.

Basándose en estas investigaciones DRACH (3) estableció en 1978 la clasificación de las prostatitis (Tabla I) que oficialmente ha perdurado hasta 1995, momento en que se ha sido sustituida por la patrocinada por el $\mathrm{NIH}$ (más concretamente por las sección del Nacional Institute of Health que se ocupa de la diabetes y enfermedades digestivas y renales) (4-6). En la clasificación de DRACH, la prostatitis crónica se califica de bacteriana o no bacteriana y se añade el término de prostatodinia, término que cobija un variado grupo de pacientes en los que dominan las incomodidades, molestias y dolores perineales (7), asociadas o no a trastornos miccionales, en los que los hallazgos analíticos son la mayor parte de las

TABLA II. PROSTATITIS

POBLACIONES MICROBIANAS AISLADAS

\begin{tabular}{|c|c|}
\hline AGUDA & CRÓNICA \\
\hline $\begin{array}{l}\text { Patógenos frecuentes: } \\
\text { E. Coli } \\
\text { Proteus mirabilis } \\
\text { Klebsiella spp. } \\
\text { P. aeruginosa } \\
\text { Enterococcus spp. } \\
\text { S. aureus' } \\
\text { Patógenos raros: } \\
\text { Enterobacter spp. } \\
\text { Serratia spp. } \\
\text { Salmonella spp. } \\
\text { Anaerobios } \\
\text { N. gonorrhoeae } \\
\text { Mycobacterium tuber- } \\
\text { culosis } \\
\text { Clostridium spp. } \\
\text { Hongos } \\
\text { Parásitos }\end{array}$ & $\begin{array}{l}\text { Patógenos reconocidos: } \\
\text { - frecuentes: } \\
\text { E. Coli (80\%) } \\
\text { Klebsiella spp. } \\
\text { Proteus mirabilis } \\
\text { Otras Enterobacteriaceae } \\
\text { P. aeruginosa } \\
\text { Enterococcus faecalis } \\
\text { - raros: } \\
\text { Candida spp. } \\
\text { Blastomyces dermatitidis } \\
\text { Histoplasma capsulatum } \\
\text { Cryptococcus neoformans } \\
\text { Mycobacterium tuberculosis } \\
\text { Mycobaccterium spp. } \\
\text { Organismos de significado } \\
\text { duduso: } \\
\text { Staphylococcus spp. } \\
\text { Corynebacterium spp. } \\
\text { Chlamydia trachornatis } \\
\text { Ureaplasma urealitycum } \\
\text { Mycoplasma hominis }\end{array}$ \\
\hline
\end{tabular}

veces irrelevantes y dudosamente motivados por una patología específicamente prostática. En la nueva clasificación se introducen variaciones conceptuales que no necesariamente parecen pertinentes desde una perspectiva crítica. Se mantienen los términos de prostatitis aguda, crónica bacteriana o inflamatoria, que tienen su correspondencia con la terminología previa, pero se da un protagonismo especial al dolor pélvico, sea cual sea su origen y motivación (síndrome prostatitis crónica-dolor pélvico) y se introduce un cuarto término que corresponde a una definición histológica y no anatomoclínica, que ciertamente podemos calificar de "prostatitis histológica", que ha de ser considerado con las mayores reservas.

El protagonismo concedido al dolor pélvico, que es el síntoma dominante en un grupo notable de estos pacientes (7), ha instalado la terminología de la prostatitis crónica en un campo aún más confuso, de límites mucho más imprecisos que el de prostatodinia (8). Por otro lado, la relevancia sintomática dolorosa ha justificado, además de una exigencia más rigurosa en la historia clínica de estos enfermos, un nuevo baremo sintomático que también ha sido motivo de aprobación general e incluso convalidación al español (puertorriqueño) (9). Diversos estudios han demostrado que la localización más frecuente del dolor en los pacientes con síndrome de prostatitis-dolor pélvico, se refiere a las siguientes áreas; próstata y área perineal $(46 \%)$, escroto y testículos $(39 \%)$, pene $(6 \%)$, vejiga $(6 \%)$, área lumbar baja $(2 \%)$ (Zermann y cols. 1999) (10). Los síntomas urinarios que más frecuentemente acompañan al dolor son los siguientes: aumento de la frecuencia miccional, dificultad para iniciar la micción, chorro débil, micción intermitente con esfuerzo o dolor en el área uretral al orinar o escozor (Alexander y cols. 1996) (11).

El nuevo término que se propone, en el apartado IV, de prostatitis asintomática inflamatoria, esta distante de la observación clínica e intenta introducir en esta variada clasificación de las prostatitis una observación histológica caracterizada por la presencia de células mononucleares (no polimorfos!) en las biopsias que habitualmente practicamos en enfermos con sospecha de cáncer de próstata o por otros motivos. Este es el grupo más importante de población que sustenta esta nueva terminología.

\section{Dualidad prostática y prostatitis crónicas}

Es interesante constatar que rara vez se ha intentado determinar qué parte de la glándula prostática, la próstata craneal o caudal, es la que sufre con más frecuencia los episodios infectivos o simplemente inflamatorios. Hay evidencias para sospechar que la 
prostatitis aguda, en la población adulta con edad inferior a los 50 años, antes del desarrollo de la hiperplasia prostática benigna (HBP), es una enfermedad de la próstata caudal (12). Algunos argumentos a favor de esta hipótesis serían los siguientes: desde el punto de vista anatómico el drenaje de la próstata caudal es mucho más directo que el de la próstata craneal permitiendo con más facilidad el reflujo, si es que este proceso facilita la infección; en la prostatitis aguda, flemonosa, se reconoce muy bien al tacto la dureza de la próstata caudal y cuando el proceso progresa hasta constituir un absceso, suele perforarse por vía rectal, cosa que serían menos frecuente si la afección se localizará en la próstata craneal, con un drenaje más fácil en uretra; finalmente, la próstata caudal es el lugar selectivo de la litiasis prostática, con frecuencia relacionada con procesos infectivos crónicos glandulares (12-13).

La pregunta de mayor trascendencia en la actualidad es la siguiente: jes la prostatitis crónica una enfermedad específica de la próstata caudale. Las implicaciones de esta pregunta no son baladíes desde el momento en que la inflamación crónica ha sido relacionada con la híperplasia prostática benigna y el carcinoma prostático. En relación con la híperplasia prostática benigna es un hallazgo relativamente frecuente encontrar infiltrados de células mononucleares en el tejido obtenido por resección transuretral o adenomectomia retropúbica (14). De hecho, este hallazgo casi constituye un fenómeno histológico universal. La identificación histológica de ésta población celular ha confirmado que está fundamentalmente formada por linfocitos, con dominios topográficos específicos en favor de linfocitos $T$ (nódulos inflamatorios) o linfocitos $B$ (infiltración intersticial). Ulteriores investigaciones han demostrado que los linfocitos enriquecen el ambiente celular con la presencia de numerosos marcadores inflamatorios (prostaglandinas, leucotrienos, factores de crecimiento, etc.) (14-15) sugiriendo que ésta población celular tiene una participación activa en los mecanismos de progresión de la hiperplasia, sin duda en coincidencia sinergística con factores hormonales. No obstante, es éste un proceso muy complejo en el que aún faltan eslabones substanciales y evidencias que den a esta hipótesis mofogénica de la HBP argumentos sólidos.

La interrelación entre inflamación prostática y carcinoma prostático ha sido sugerida y defendida por el grupo de De Marzo (16) que incluso han presentado una secuencia patogénica, en la que se implican hallazgos histológicos específicos, como sería la lesión calificada como PIA (postinflamatory atrophy), que precedería al PIN en su evolución hacia el carcinoma prostático. Es de suponer, desde el punto de vista conceptual, que esta lesión se presentaría fundamentalmente en la próstata caudal, en la zona en la que se originan más del $80 \%$ de los carcinomas prostáticos, pero no es este un aspecto claramente reconocido en la literatura. Por el contrario, nosotros hemos buscado esta lesión en el tejido perteneciente al adenoma, en muestras obtenidas por RT o adenomectomia retropúbica, y no hemos sido capaces de identificarla en ningún caso (Manzarbeitia y cols. 2005) (16).

\section{Diagnóstico de la prostatitis crónica}

El diagnóstico de la prostatitis crónica requiere el sustento patogénico de la presencia de bacterias o células inflamatorias en la glándula prostática. Los instrumentos empleados para esta necesaria demostración no son muchos y se refieren fundamentalmente a la prueba propuesta por STAMEY-MEARES, previamente descrita. Asumiendo que la prueba se realiza con el mayor rigor, obteniéndose un importante volumen de secreción prostática, nuevamente en este caso ignoramos de donde procede esta secreción, si de la próstata caudal, craneal, o de ambas, aunque sospechamos que la mayor parte debe tener origen en la próstata caudal, la parte de la glándula más directamente en contacto con el dedo de explorador. A partir de las muestras obtenidas, realizados los cultivos y las observaciones al microscopio con los contajes bacterianos y de leucocitos correspondientes, la interpretación de los resultados no es fácil. Si la cantidad de bacterias es al menos diez veces superior en las últimas muestras que en las muestras de orina iniciales (M1-M2), previas al masaje prostático, se considera que la infección está localizada en la próstata. $\mathrm{Si}$ el número de leucocitos es claramente superior (10/15 por campo) en las muestras postmasaje, junto a otros hallazgos sugestivos de inflamación (reducción gotas lipoideas, cuerpos amiláceos o abundantes leucocitos, etc.) (18) se sospechará que existe inflamación prostática.

No existen bacterias típicas o específicamente productoras de prostatitis crónica, al menos en el momento actual (19-20). Las bacterias más frecuentemente encontradas en la secreción prostática son enterobacterias de escasa especificidad (Tabla II). Con frecuencia se plantea la controversia sobre su auténtico carácter patogénico y su participación en el proceso que sufre el enfermo. Tampoco la sospecha de microorganismos más específicos, pero más difícilmente identificables, como se ha propuesto en los últimos años, ha sido confirmada (21).

En relación con la población celular inflamatoria reconocida en la secreción prostática que 
caracteriza a la prostatitis crónica, tampoco existe ningún dato de especificidad o correlación con los síntomas (22). Se ha buscado una mayor especificidad en algunas proteínas presentes en la secreción prostática obtenida tras el masaje; De este modo se ha investigado la presencia de IL-1, TNF $\alpha$, y otros muchos marcadores con resultados orientativos, no definitivos (23-25) (Tabla II). También podría utilizarse con estos fines el nivel plasmático del PSA que aumenta coincidiendo con los procesos infectivos, a veces en proporciones muy elevadas (25).

En definitiva las pruebas que constituyen el fundamento diagnóstico de la prostatitis crónica carecen de especificidad, igual que sucede con el perfil clínico de estos pacientes. De hecho, el diagnóstico de prostatitis crónicas es, en muchas ocasiones, un diagnóstico de exclusión. Más de un $30 \%$ de los pacientes que calificamos de prostatitis crónica se quedan sin amparo etiopatogénico concluyente (25-26). La tendencia actual es simplificar los procedimientos diagnósticos de tal modo que la prueba de STAMEY-MEARES queda reducida sólo a dos muestras: la muestra de orina obtenida inmediatamente antes del masaje y la muestra obtenida después del masaje prostático, aunque el algoritmo diagnóstico que propone la EAU (26), aún incluye la prueba original de Meares y Stamey. La ecografía transrectal constituye, desde que es disponible y se ha divulgado, un recurso frecuente de estudio en estos pacientes y ha producido hallazgos de difícil sistematización, referidos a la complejidad venosa periprostática o al mismo cuello vesical (di Trapani y cols. 1988, Dellabella y cols. 2006) (27-28). Ciertamente, el procedimiento que aportaría mayor información sobre la situación inflamatoria de la glándula prostática sería la biopsia prostática perineal que por ahora sólo parece indicada en pacientes en los que se sospecha la coincidencia de cáncer prostático por PSA elevado, o hallazgos de induraciones sospechosas al tacto rectal. Debe recordarse, aunque infrecuente, que la prostatitis granulomatosa es una entidad anatomoclínica perfectamente definida en la que la biopsia prostática es incuestionable; He aquí una nueva ausencia en la clasificación de la prostatitis.

\section{Prostatitis crónica y dolor pélvico}

El dolor es sin duda el síntoma dominante en muchos pacientes a los que se les diagnostica de prostatitis crónica $(7,25)$. Como señalado, el dolor se refiere fundamentalmente a la zona pélvica, área perineal

TABLA III. CITOQUINAS COMO MARCADORES BIOQUIMIICOS

DE INFLAMACIÓN EN PLASMA SEMINAL O SECRECIÓN PROSTÁTICA*

\begin{tabular}{|c|c|c|c|c|}
\hline MARCADORES & PRODUCTO & $\begin{array}{c}\text { № } \\
\text { CASOS }\end{array}$ & AUTOR & COMENTARIOS \\
\hline $\begin{array}{l}\text { NGF, } I_{6}, I_{8} \\
I F N-\gamma, I_{2}, I_{10}\end{array}$ & Seminal & 31 & Miller y cols. 2002 & $\begin{array}{l}\text { NGF y citoquinas pueden tener un } \\
\text { papel en el dolor de los pacientes en } \\
\text { PC-DP }\end{array}$ \\
\hline $\begin{array}{l}\mathrm{IL}_{8}, \mathrm{IFN}-\gamma \\
\mathrm{IL}_{2}, \mathrm{IL}_{10}\end{array}$ & Seminal & 31 & Miller y cols. 2002 & $\begin{array}{l}\text { Significativamente aumentados en los } \\
\text { pacientes con PC-DP }\end{array}$ \\
\hline $\mathrm{IL}_{10}, \mathrm{IL}_{8}$ & $\begin{array}{l}\text { Secreción } \\
\text { prostática (masaje) }\end{array}$ & 29 & LI H. 2004 & Aumentada en pacientes sintomáticos \\
\hline TNF- $\alpha, I L 1 \beta$ & $\begin{array}{l}\text { Secreción prostática } \\
\text { (masaje) }\end{array}$ & 78 & Nadler y cols. 2000 & $\begin{array}{l}\text { Diferente aumento en PC IIla y IIIb así } \\
\text { como entre III y IV }\end{array}$ \\
\hline TNF- $\alpha, I L 1 \beta$ & $\begin{array}{l}\text { Secreción prostática } \\
\text { (masaje) }\end{array}$ & 34 & Yang, J. 2004 & $\begin{array}{l}\text { Aumentados en pacientes con leuco- } \\
\text { citaria }\end{array}$ \\
\hline $\mathrm{IL}_{6} \mathrm{IL}_{8}$ & Seminal & 207 & Paulis, G. 2003 & $\begin{array}{l}85 \% \text { de los casos aumentada en com- } \\
\text { paración con casos control }\end{array}$ \\
\hline
\end{tabular}

* tomado de McConnell y cols. 2006 
y región prostática. El baremo sintomático empleado y recomendado por el $\mathrm{NIH}$, incluye al menos tres preguntas relacionadas con el dolor, reconociendose la importancia de éste tema en la historia natural de las llamadas prostatitis crónicas. Habitualmente el síndrome doloroso se acompaña de una enorme carga de ansiedad, que no es investigada en dicho baremo.

Un aspecto absolutamente trascendental, y en el que se ha avanzado bastante en los últimos meses, está relacionado con el origen e interpretación del dolor pélvico. Los expertos en patología dolorosa reconocen que puede haber cuatro factores diferentes que motiven este tipo de dolor, clasificados del siguiente modo: nociceptivo, inflamatorio, neuropático y disfuncional (29). Cada uno de estos mecanismos tiene factores desencadenantes distintos y patogenia diferente que ha sido recientemente investigada con la intención de encontrar nuevas estrategias terapéuticas. En algunos de estos pacientes, el dolor insufrible y recalcitrante supera cualquier iniciativa terapéutica imaginable. En nuestra experiencia tenemos que destacar la psicopatía, abierta o oculta, que existe en muchos de estos pacientes, algunos calificados por los psiquiatras como síndrome bipolar y otras enfermedades afines.

El análisis riguroso del dolor pélvico orienta cada día más en el sentido de que el protagonismo de la próstata no es tan importante y que son otros factores los que pueden motivarlo. Por esta razón se investiga actualmente la posibilidad de un tratamiento con analgésicos y opiáceos, más que con la clásica tríada terapéutica de las tres a: antimicrobianos, antiinflamatorios y alfabloqueantes, a los que habría que añadir, porque en algunos casos se han considerado como extremadamente útiles, antidepresivos y ansiolíticos $(25,26,29)$.

El papel del dolor pélvico en la prostatitis crónica puede indagarse investigando el perfil clínico de los enfermos incluidos en los ensayos clínicos que se han realizado hasta la fecha, con cierto rigor, incluyendo comparación con placebo o doble ciego (25-26). Puede comprobarse que el perfil clínico de estos enfermos que no es muy homogéneo, ni en la edad. Por lo tanto, es difícil alcanzar conclusiones convincentes sobre la eficacia terapéutica de los diversos programas propuestos ya que sus resultados dependen, en un alto grado, del tipo de enfermo que se incluyó en dicha investigación. La conclusión más evidente de estos estudios es que hay un porcentaje importante de enfermos con el síndrome de prostatitis crónica-dolor pélvico que escapa a cualquier iniciativa terapéutica tradicional y justifica sobradamente la búsqueda de nuevas estrategias terapéuticas.

\section{Prostatitis histológica y su inclusión en la clasificación de las prostatitis.}

La presencia de elementos mononucleares en el tejido prostático (adenoma), en muestras obtenidas por muy variados métodos, pero fundamentalmente en las muestras de resección transuretral y adenomectomía retropúbica, es un hallazgo universal, de mayor o menor intensidad, reconocido desde hace muchos años. En el caso concreto de la HBP numerosos estudios, ya señalados, han investigado el significado patológico de esta inflamación y su potencial participación en la génesis de la HBP. El tema ha recibido una mayor atención desde el momento en que se ha involucrado la inflamación en la génesis del cáncer de próstata. Estudios más recientes confirman que en los individuos en los que la biopsia de próstata se realiza por sospecha de cáncer prostático, que tienen inflamación, la posibilidad de un cáncer de próstata a cinco años es estadísticamente más alta que en los que no tenían inflamación (McLennan y cols. 2006) (30).

Todo este tema es extremadamente interesante tanto desde el punto de vista biológico como patológico pero no parece existir ningún motivo, desde la perspectiva anatomoclínica, no exclusivamente histológica, que justifique la inclusión de estos casos en un nuevo grupo de "prostatitis crónica" como propuesto en la clasificación del $\mathrm{NIH}$. Además, independientemente de que la inflamación pueda afectar a la zona de transición o a la próstata caudal, ciertamente cuando afecta al "adenoma" en rigor, tendríamos que referirnos a un "adenomitis".

\section{CONCLUSIONES}

Los extraordinarios esfuerzos realizados en los últimos años para identificar la etiología, patogenia y perfil clínico de los pacientes con "prostatitis crónica" y de este modo conseguir una clasificación de contenidos nosológicos anatomoclínicos y microbiológicos, de aplicación rigurosa en la práctica clínica, permiten una visión crítica de la clasificación actual de las "prostatitis crónicas"; El síndrome de prostatitis crónica-dolor pélvico, incluido en el apartado III de la clasificación NIH, cada día realza mas el dolor pélvico y gana más argumentos a favor de un dolor no necesariamente de origen prostático, justificando nuevas opciones terapéuticas directamente relacionadas con el dolor pélvico y no con la próstata o su inflamación. Por otro lado, el concepto de "prostatitis histológica", incluido en el apartado IV del NIH debe ser eliminado de esta clasificación e identificado con un nuevo título más relevante y acorde con los más recientes progresos relacionando el papel de la inflamación crónica con la carcinogénesis prostática o con la hiperplasia prostática multinodular (HBP). 


\section{BIBLIOGRAFÍA y LECTURAS RECOMENDADAS ( ${ }^{*}$ lectura de interés $y^{* *}$ lectura fundamental)}

**1. STAMEY, T.: "Urinary infections". William and Wilkins, Baltimore, 1972.

**2. MEARES, E.M.; STAMEY, T.A.: "Bacteriologic localization patterns in bacterial prostatitis and urethritis". Invest. Urol., 5: 492, 1968.

**3. DRACH, G.W.; FAIR, W.R.; MEARES, E.M. y cols.: "Classification of benign diseases associated with prostatic pain: prostatitis or prostatodynia?" J. Urol., 120: 266, 1978.

**4. INTERNATIONAL CLASSIFICATION OF DISEASES (ICD).: 10th version. Geneva, Who, 1989.

**5. WORKSHOP COMMITTEE OF THE NATIONAL INSTITUTEOFDIABETES AND DIGESTIVEAND KIDNEY DISEASE (NIDDK):: "Chronic prostatitis workshop". Bethesda, Maryland, Dec 7-8, 1995.

6. KRIEGER, J.N.; NYBERG, L. JR.; NICKEL, J.C.: "NIH consensus definition and classification of prostatitis". JAMA, 282: 236, 1999.

7. KRIEGER, J.N.; EGAN, K.J.; ROSS, S.O. y cols.: "Chronic pelvic pains represent the most prominent urological symptoms of "chronic prostatitis". Urology, 48: 715, 1996.

8. KRIEGER, J.N.; JACOBS, R.R.; ROSS, S.O.: "Does the chronic prostatitis/pelvic pain syndrome differ from nonbacterial prostatitis and prostatodynia?" J. Urol., 164: 1554, 2000.

9. LITWIN, M.S.; McNAUGHTON-COLLINS, M.; FOWLER, F.J. JR. y cols.: "The National Institute of Health chronic prostatitis symptom index: development and validation of new outcome measure. Chronic Prostatitis Collaborative Research Network”. J. Urol., 162: 369, 1999.

10. ZERMANN, D.H.; ISHIGOOKA, M.; DOGGWEILER, R. y cols.: "Neurourological insights into the etiology of genitourinary pain in men". J. Urol., 161: 903, 1999.

11. ALEXANDER, R.B.; TRISSEL, D.: "Chronic prostatitis: Results of an internet survey". Urology, 48: 568, 1996.

*12. GIL VERNET, S.: "Enfermedades de la prostate". Tomo II, Vol II Editorial Paz Montalvo Madrid, 1955.

*13. CIFUENTES DELATTE, L.: "Cirugía Urológica Endoscópica”. Ed. Paz Montalvo (2 edición) Madrid, 1981.

14. VELA NAVARRETE, R.; GARCÍA CARDOSO, J.V.; BARAT, A. y cols.: "BPH and inflammation: pharmacological effects of Permixon on histological and molecular inflammatory markers. Results of a double blind pilot clinical assay". Eur. Urol., 44: 549, 2003.

15. VELA NAVARRETE, R.; ESCRIBANO BURGOS, M.; FARRE, A.L. y cols.: "Serenoa Repens treatment modifies bax-bcl-2 index expresión and caspase-3 activity in prostatic tissue from patients with benign prostatic hyperplasia”. J. Urol., 173: 507, 2005.
*16. DE MARZO, A.M.; MARCHI, V.L.; EPSTEIN, J.I. y cols.: "Proliferative inflammatory atrophy of the prostate: implications for prostatic carcinogenesis". Am. J. Pathol., 155: 1985, 1999.

17. MANZARBEITIA, F.; VELA NAVARRETE, R.; SARASA, J.L. y cols.: "BPH: Histological aspects of adenoma mononuclear cell infiltration". Eur. Urol. Suppl., 5: 123, 2006.

18. CIFUENTES DELATTE, L.: "El laboratorio del urólogo". Salvat, Barcelona, 1974.

19. SCHAEFFER, A.J.: "Prostatitis: US perspectiva". Int. J. Antimicrob. Agents, 11: 205, 1999.

20. SCHNEIDER, H.; LUDWIG, M.; HOSSAIN, H.M. y cols.: "The 2001 Giessen Cohort Study on patients with prostatitis syndrome an evaluation of inflammatory status and search formicroorganisms 10 years after a first analysis". Andrologia, 35: 258, 2003.

21. BADALYAN, R.R.; FANARJYAN, S.V.; AGHAJANYAN, I.G.: "Chlamydial and ureaplasmal infections in patients with nonbacterial chronic prostatitis". Andrologia, 35: 263, 2003.

22. SCHAEFFER, A.J.; KNAUSS, J.S.; LANDIS, J.R. y cols.: "Chronic Prostatitis Collaborative Research Network Study Group. Leucocyte and bacterial counts do not correlate with severity of symptoms in men with chronic prostatitis: the National Institutes of Health Chronic Prostatitis Cohort Study". J. Urol., 168: 1048, 2002.

*23. ALEXANDER, R.B.; PONNIAH, S.; HASDAY, J. y cols.: "Elevated levels of proinflammatory cytokines in the semen of patients with chronic prostatitis/chronic pelvic pain syndrome". Urology, 52: 744, 1998.

*24. NADLER, R.B.; KOCH, A.E.; CALHOUN, E.A. y cols.: "IL-1beta and TNF-alpha in prostatic secretions are indicators in the evaluation of men with chronic prostatitis". J. Urol., 164: 214, 2000.

25. McCONNELL, J.; ABRAMS, P.; KHOURY, S. y cols.: "Male lower urinary tract dysfunction". Edition 2006.

**26. EAU.: "Prostatitis and Chronic pelvic pain syndrome". Guidelines, pag 89-95, 2006.

27. DI TRAPANI, D.; PAVONEC, C.; SERRETA, V. y cols.: "Chronic prostatitis and prostatodynia: uretrasonographic alterations of the prostata bladder neck seminal vesicles and periprostatic venous plexus". Eur. Urol., 15: 230, 1988.

28. DELLABELLA, M.; MILANESE, G.; MUZZONIFRO, G.: "Correlation between ultrasounds alterations of the preprostatic sphincter and symptoms in patients with chronic prostatitis-chronic pelvic pain syndrome". J. Urol., 176: 112, 2006.

**29. NICKEL, J.C.: "Opiods for chronic prostatitis and intersticial cystitis: lessons learned from the 11th World Congress on pain". Urology, 68: 697, 2006.

30. MACLENNAN, G.T.; EISENBERG, R.; FLESHMAN, R.L. y cols.: "Does chronic inflammation influence prostate carcinogenesis? A five year follow up study”. J. Urol., 175: 35, 2006. 